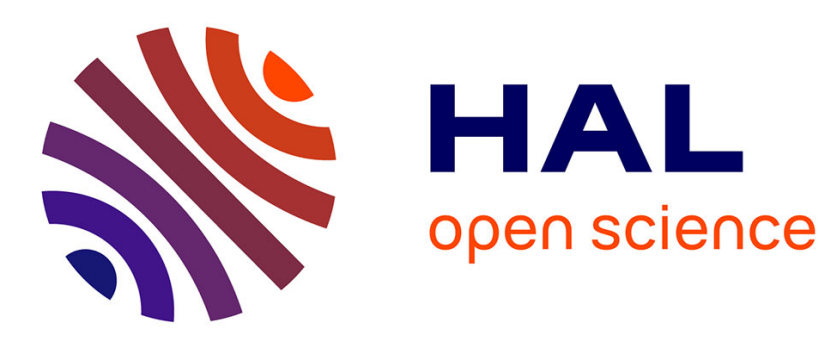

\title{
Fault diagnosis for linear time-varying descriptor systems
}

Abdouramane Moussa Ali, Qinghua Zhang

\section{To cite this version:}

Abdouramane Moussa Ali, Qinghua Zhang. Fault diagnosis for linear time-varying descriptor systems. 11th IFAC International Workshop on Adaptation and Learning in Control and Signal Processing, Jul 2013, Caen, France. pp.724-729, 10.3182/20130703-3-FR-4038.00073 . hal-00988297

\section{HAL Id: hal-00988297 https://hal.science/hal-00988297}

Submitted on 7 May 2014

HAL is a multi-disciplinary open access archive for the deposit and dissemination of scientific research documents, whether they are published or not. The documents may come from teaching and research institutions in France or abroad, or from public or private research centers.
L'archive ouverte pluridisciplinaire HAL, est destinée au dépôt et à la diffusion de documents scientifiques de niveau recherche, publiés ou non, émanant des établissements d'enseignement et de recherche français ou étrangers, des laboratoires publics ou privés. 


\title{
Fault diagnosis for linear time-varying descriptor systems
}

\author{
Abdouramane Moussa Ali* Qinghua Zhang* \\ * Project-team SISYPHE of INRIA, Campus de Beaulieu, \\ 35042 Rennes, France \\ (e-mail: \{abdouramane.moussa_ali, qinghua.zhang@\}@inria.fr)
}

\begin{abstract}
In this paper fault diagnosis is studied for linear time varying descriptor systems, which are the discrete time counterpart of the continuous time dynamic systems described by differentialalgebraic equations. This class of systems includes and is broader than the well-known state space systems. The framework of descriptor systems is particularly useful for studying dynamic systems exhibiting time varying singularities. Actuator faults and sensor faults are respectively modeled as parametric changes in the state equation and in the output equation. The main result of this paper consists in extending an adaptive observer, initially designed for state space systems, to descriptor systems. Based on this result, fault diagnosis is performed by estimating the parameters characterizing actuator and sensor faults. Simulations examples are presented to illustrate the proposed method.
\end{abstract}

Keywords: fault diagnosis, discrete-time descriptor dynamics, time-varying models, adaptive observer.

\section{INTRODUCTION}

Many modern engineering systems can be modeled by an explicit ordinary differential equation (ODE) of the form

$$
\dot{x}(t)=f(x(t), u(t))
$$

where $x(t)$ and $u(t)$ represent respectively the (vectorial) state and input of the system, $\dot{x}(t)$ denotes the time derivative of $x(t)$, and $f(\cdot, \cdot)$ is some function characterizing the system. Such state space equations have a long-term mathematical history, and a large number of analytical and numerical tools have been developed for their study.

However, in some cases such an explicit state space model for the dynamics of a given system is not available. The system may instead be described by implicit differential equations, known as differential-algebraic equations (DAE), of the form

$$
F(\dot{x}(t), x(t), u(t))=0
$$

where $F(\cdot, \cdot, \cdot)$ is some vector-valued function. If $\dot{x}(t)$ can be solved for from (2), then the DAE can be converted to an ODE, but this operation is not always possible. It is thus necessary to study DAE systems in some situations.

After linearization around an operating point and discretization in time, the original nonlinear DAE system is approximately described by an implicit discrete time state space equation

$$
E_{k+1} x(k+1)=A_{k} x(k)+B_{k} u(k)+\mu(k)
$$

where $x(k), u(k)$ and $\mu(k)$ are respectively the discrete time state, the input and the modeling errors indexed by $k=0,1,2, \ldots$, and the matrix $E_{k}$ may not be of full column rank.

\footnotetext{
^ This work was supported by the ITEA2 MODRIO project.
}

Systems governed by (3) are known as descriptor systems. This is a general and convenient framework for studying DAE systems which can be appropriately linearized.

Some descriptor systems can be simply regarded as implicitly written state-space equations, this is the case of systems in which the matrix $E_{k}$ has full column rank. In principle, the theory developed in the framework of linear state-space equations can be applied in this case. However, even if the matrix $E_{k}$ is invertible for all $k$, it is preferable to use the descriptor equation because of the possible ill conditioning of $E_{k}$.

Descriptor systems have attracted considerable attentions in recent decades where great efforts where made to investigate descriptor system theory and applications (Nikoukhah et al. (1992), Benveniste et al. (1993), Darouach and Boutayeb (1995), Shields (1997), Polycarpou et al. (1997), Zhang et al. (1998), Vemuri et al. (2001), Koenig and Mammar (2002)).

Fault diagnosis (detection and identification of fault) is rarely tackled in the descriptor case, in contrast to the case of systems with classical state space representations, where the theory is well-established (Ding (2008), Isermann (2006) and references therein). For descriptor system fault diagnosis, most studies are observer-based approaches in the time invariant case (Duan et al. (2002), Marx et al. (2004)).

In this paper, fault diagnosis will be studied for the general class of time-varying discrete time linear descriptor systems in the form of 


$$
\begin{aligned}
E_{k+1} x(k+1) & =A_{k} x(k)+B_{k} u(k)+\Phi_{k} \theta+\mu(k) \\
y(k+1) & =C_{k+1} x(k+1)+\Psi_{k} \theta+\nu(k)
\end{aligned}
$$

$$
k=0,1,2, \cdots
$$

where $x(k) \in \mathbb{R}^{n}$ is the state vector, $u(k) \in \mathbb{R}^{m}$ is the input vector (possibly including other known variables), $y(k) \in \mathbb{R}^{p}$ is the output vector, $E_{k+1}, A_{k}, B_{k}, C_{k}$ are known real matrices of compatible sizes, $\mu(k)$ and $\nu(k)$ represent modeling/measurement errors and are modeled as independent, zero-mean, Gaussian vector sequences with covariance matrices $Q_{k}$ and $R_{k}$ respectively, the terms $\Phi_{k} \theta$ and $\Psi_{k} \theta$ represent respectively faults possibly affecting the state and the output equation. The matrices $\Phi_{k} \in \mathbb{R}^{n \times q}$ and $\Psi_{k} \in \mathbb{R}^{n \times q}$ are assumed known, whereas the parameter vector $\theta \in \mathbb{R}^{q}$ is unknown and subject to changes caused by faults. The fault-free system is characterized by the nominal value $\bar{\theta}$ of the parameter vector.

The development of this paper is based, on the one hand, on the adaptive estimation techniques for explicit state space systems (Zhang (2002)), and, on the other hand, on the Kalman filter for time-varying discrete linear descriptor systems. The core of the proposed fault diagnosis methods consists of an adaptive observer which is used both to estimate the monitored faults and to improve the robustness against model uncertainty due to parameter changes.

The paper is organized as follows. The first step in the monitoring of a system modeled by (4) is necessarily the state space $x$ reconstruction when the only information available is contained in signals $u$ and $y$. In Section 2, under some assumptions, a transformation of descriptor systems is introduced which allows us to use a standard Kalman filter for state vector estimation, in the fault-free case. Section 3 is devoted to the outline of the approach discussed in this paper. We detail the motivations of this contribution and explain how adaptive observer based fault diagnosis approach can be extended to general linear descriptor systems. In section 4, a simulation example is used to illustrate the effectiveness of the proposed approach.

\section{KALMAN FILTER FOR FAULT-FREE DESCRIPTOR SYSTEMS}

Here we recall some basic knowledge about the Kalman filter of discrete linear descriptor systems described by

$$
\begin{gathered}
E_{k+1} x(k+1)=A_{k} x(k)+B_{k} u(k)+\mu(k) \\
y(k+1)=C_{k+1} x(k+1)+\nu(k) \\
k=0,1,2, \cdots
\end{gathered}
$$

Like the classical Kalman filter for state space systems, the purpose of descriptor system Kalman filter is for state estimation from input and output signals. State reconstruction has been frequently studied in continuous time and usual for state space systems case (when $E=I$ ) since the early work in (Kalman (1960), Kreindler and Sarachik (1964), Silverman and Meadows (1967)) and in the time invariant descriptor case. Theoretical work on the observability of linear time-varying continuous time descriptor systems can be found in (Campbell and Terrell (1991)).
In (Nikoukhah et al. (1992)), the authors developed a Kalman filter for (5), based on maximum likelihood estimation. The filtered estimate $\hat{x}$ is defined as the maximum likelihood estimate of $x$ based on (5) together with a priori knowledge of the initial condition $x(0)$. Recursively, we obtain, from (5) and the estimate of $x(k)$ denoted by $\hat{x}(k)$, the following equation

$$
Y_{k}=H_{k+1} x(k+1)+V_{k}, \quad k=0,1, \cdots
$$

where

$$
Y_{k}=\left[\begin{array}{c}
A_{k} \hat{x}(k)+B_{k} u(k) \\
y(k+1)
\end{array}\right], H_{k}=\left[\begin{array}{c}
E_{k} \\
C_{k}
\end{array}\right], V_{k}=\left[\begin{array}{c}
w(k) \\
\nu(k)
\end{array}\right]
$$

$w(k)=-A_{k} \tilde{x}(k)-\mu(k)$ and $\tilde{x}(k)=x(k)-\hat{x}(k)$ is the state estimate error.

It has been shown in (Nikoukhah et al. (1992)) (Lemma 2.3 and Lemma 2.4) that the (optimal) estimate $\hat{x}(k+1)$ of $x(k+1)$ based on $(5)$ is the same as its (optimal) estimate based on (6).

Let $\mathcal{R}_{k}$ be the covariance matrix of $V_{k}$. It is shown in (Nikoukhah et al. (1992)) that

$$
\mathcal{R}_{k}=\left[\begin{array}{cc}
A_{k} P_{k} A_{k}^{T}+Q_{k} & 0 \\
0 & R_{k}
\end{array}\right]
$$

where $P_{k}$ is a symmetric positive definite matrix, which can be computed at each iteration of the maximum likelihood estimation algorithm, as recalled later in this section.

We shall make the following assumptions on system (5).

A1 : The initial state $x(0)$ is Gaussian with known mean $\bar{x}_{0}$ and variance $P_{0}$ and independent of $\mu$ and $\nu$. This can be represented by the following equation

$$
x(0)=\bar{x}_{0}+\eta
$$

where $\eta$ is zero-mean, Gaussian and independent of $\mu$ and $\nu$, and with covariance $P_{0}$.

A2 : The matrix $H_{k}$ is supposed to be full column rank $\forall k \geq 1$

A3 : For all $k \geq 0$, the variance matrices $Q_{k}$ and $R_{k}$ are positive definite.

From (6), the maximum likelihood estimate of $x(k+1)$ consists in maximizing the probability density function of $V_{k}$ parameterized by $x(k+1)$. This estimate is given by

$$
\hat{x}(k+1)=\arg \max _{\alpha} e^{\left\{-\frac{1}{2}\left(Y_{k}-H_{k+1} \alpha\right) \mathcal{R}_{k}^{-1}\left(Y_{k}-H_{k+1} \alpha\right)\right\}}
$$

The solution of the maximization in (8) gives

$$
\hat{x}(k+1)=\left(H_{k+1}^{T} \mathcal{R}_{k}^{-1} H_{k+1}\right)^{-1} H_{k+1}^{T} \mathcal{R}_{k}^{-1} Y_{k}
$$

The error covariance associated with the estimate $\hat{x}(k+1)$ is

$$
P_{k+1}=\left(H_{k+1}^{T} \mathcal{R}_{k}^{-1} H_{k+1}\right)^{-1}
$$

Note that, the inverse matrices in (9) and (10) are well defined because of assumptions A2 and A3.

Consider the following partition

$$
\left[\begin{array}{ll}
L_{k} & K_{k}
\end{array}\right]=P_{k+1} H_{k+1}^{T} \mathcal{R}_{k}^{-1}
$$

The estimate $\hat{x}(k+1)$ can be explicitly expressed from (6), (9) and (11), yielding

$$
\hat{x}(k+1)=L_{k} A_{k} \hat{x}(k)+K_{k} y(k+1)+L_{k} B_{k} u(k)
$$


and the state estimation error is then governed by the equation

$$
\tilde{x}(k+1)=L_{k} A_{k} \tilde{x}(k)+L_{k} \mu(k)-K_{k} \nu(k)
$$

Let $\tilde{P}_{k}$ be the predicted estimate covariance :

$$
\tilde{P}_{k}=A_{k-1} P_{k} A_{k-1}^{T}+Q_{k-1}
$$

The following Riccati equation is obtained from (10)

$$
\begin{gathered}
\tilde{P}_{k+1}=A_{k}\left(E_{k+1}^{T} \tilde{P}_{k}^{-1} E_{k+1}+C_{k+1}^{T} R_{k}^{-1} C_{k+1}\right)^{-1} A_{k} \\
+Q_{k}
\end{gathered}
$$

We know that the stability of the Kalman filter (the optimal estimator) is generally guaranteed if the covariance matrix $\tilde{P}_{k}$ is bounded from above and from below by two strictly positive definite matrices for all $k \geq 0$.

In (Nikoukhah et al. (1992)), the asymptotic behavior of the error variance $P_{k}$ was examined in the time invariant case (with constant matrices $A, C, E, Q, R$ ). It can be shown in this case that this error variance $\tilde{P}_{k}$ converges exponentially fast to the unique positive definite solution of the algebraic descriptor Riccati equation (Nikoukhah et al. (1987)):

$$
\tilde{P}=A\left(E^{T} \tilde{P}^{-1} E+C^{T} R^{-1} C\right)^{-1} A^{T}+Q
$$

\section{ADAPTIVE OBSERVER}

Consider the system in (4) subject to actuator and sensor faults. By defining $L_{k}$ and $K_{k}$ as in (11), the following explicit state recurrent equation is derived from (4):

$$
\begin{aligned}
x(k+1)= & L_{k} A_{k} x(k)+K_{k} y(k+1)+L_{k} B_{k} u(k) \\
& +\left[L_{k} \Phi_{k}-K_{k} \Psi_{k}\right] \theta+L_{k} \mu(k)-K_{k} \nu(k) \\
y(k+1)= & C_{k+1} x(k+1)+\Psi_{k} \theta+\nu(k) \\
& k=0,1,2, \cdots
\end{aligned}
$$

We assume, in addition of assumptions A1 - A3, that

A4 : $E_{k}, A_{k}, B_{k}, C_{k}, \Phi_{k}, \Psi_{k}$ are all known and bounded A5 : the parameter vector $\theta$ is constant

In fault diagnosis, the terms $\Phi_{k} \theta$ and $\Psi_{k} \theta$ can be used to model faults. In particular, they can be used to model actuator or sensor faults according to their location in the system model.

In this section, we aim to estimate the hidden variable vector $x$ and the parameter vector $\theta$ based on the knowledge of the input $u$ and the output $y$ signals. One commonly used method to solve this problem is to augment the state $x(k)$ with the parameter vector $\theta$ and to implement the Kalman filter (12). While this approach has proved effective in some applications, at least in the case of state space systems (Cox (1964)), it has also some well known drawbacks. In particular,

- treating equally the state vector $x(k)$ and the parameter vector $\theta$ as if they had similar dynamics may make the tuning of the Kalman filter delicate.

- the computational cost of the Kalman filter increases mainly because of the larger covariance matrix of the augmented state estimation error.
The approach proposed in this section relies on an adaptive observer. We extend the existing results concerning the adaptive observer for fault diagnosis in linear state space systems (Zhang (2002)) to the descriptor system.

Conceptually similar to the Kalman filter applied to the augmented system, the adaptive observer explicitly takes into account the difference between state variables and unknown parameters.

For the descriptor system described by (4) we propose the following adaptive observer

$$
\begin{aligned}
\hat{x}(k+1)= & L_{k} A_{k} \hat{x}(k)+K_{k} y(k+1)+L_{k} B_{k} u(k) \\
& +\left[L_{k} \Phi_{k}-K_{k} \Psi_{k}\right] \hat{\theta}(k)+\omega_{k} \\
\hat{y}(k+1)= & C_{k+1} \hat{x}(k+1)+\Psi_{k} \hat{\theta}(k) \\
\Upsilon_{k+1}= & L_{k} A_{k} \Upsilon_{k}+\left[L_{k} \Phi_{k}-K_{k} \Psi_{k}\right] \\
\Omega_{k+1}= & C_{k+1} \Upsilon_{k+1}+\Psi_{k} \\
\omega_{k}= & L_{k} A_{k} \Upsilon_{k}(\hat{\theta}(k)-\hat{\theta}(k-1)) \\
\hat{\theta}(k+1)= & \hat{\theta}(k)+\Theta_{k+1}(y(k+1)-\hat{y}(k+1)) \\
\Theta_{k+1}= & S_{k} \Omega_{k+1}^{T}\left(\lambda R_{k}+\Omega_{k+1} S_{k} \Omega_{k+1}^{T}\right)^{-1} \\
\Gamma_{k+1}= & \left(\lambda R_{k}+\Omega_{k+1} S_{k} \Omega_{k+1}^{T}\right)^{-1} \\
S_{k+1}= & \frac{1}{\lambda}\left(I-\Theta_{k+1} \Omega_{k+1}\right) S_{k} \\
k= & 0,1,2, \ldots
\end{aligned}
$$

where $\lambda \in(0,1)$ is a forgetting factor, $\hat{\theta}(k)$ is the estimate of the parameter vector $\theta$ at time $k$, matrices gain $L_{k}$ and $K_{k}$ are computed as in (11). The term $\omega_{k}$ added in (18a) and defined in (18e) may appear unusual. It will help to establish the proof of the convergence of this adaptive observer later in this section.

This adaptive observer is the discrete time counterpart of the continuous time algorithm presented in ( $\mathrm{Li}$ et al. (2011)). A simpler discrete time algorithm (no unknown parameter vector in the output equation) has been proposed in (Guyader and Zhang (2003)). Its generalizations to some particular class of nonlinear systems have been considered in (Xu and Zhang (2004), Zhang and Besançon (2008), Farza et al. (2009)).

The convergence of the adaptive observer (18) can be studied both in the noise-free case and in the noisecorrupted case. As the proofs are similar to those of (Guyader and Zhang (2003)) and (Li et al. (2011)), let us formulate here its main lines.

Define the state and parameter estimation errors, respectively given by

$$
\begin{aligned}
\tilde{x}(k) & =x(k)-\hat{x}(k) \\
\tilde{\theta}(k) & =\theta-\hat{\theta}(k)
\end{aligned}
$$

In the noise-free case $(\mu(k)=\nu(k)=0)$, the state estimation error is governed by the following equation derived from (17a) and (18a)

$$
\tilde{x}(k+1)=L_{k} A_{k} \tilde{x}(k)+\left[L_{k} \Phi_{k}-K_{k} \Psi_{k}\right] \tilde{\theta}(k)-\omega_{k}
$$

Define the variable

$$
z(k)=\tilde{x}(k)-\Upsilon_{k} \tilde{\theta}(k-1)
$$

From (21), (18c) and (18e), the dynamics of $z_{k}$ is simplified to an exponentially stable system 


$$
z(k+1)=L_{k} A_{k} z(k)
$$

The parameter estimation error is governed by

$$
\tilde{\theta}(k+1)=\left(I-\Theta_{k+1} \Omega_{k+1}\right) \tilde{\theta}(k)-\Theta_{k+1} C_{k+1} z(k+1)
$$

The key issue is to prove the exponential stability of the homogeneous part of (24) given by

$$
\zeta(k+1)=\left(I-\Theta_{k+1} \Omega_{k+1}\right) \zeta(k)
$$

\section{Lemma 3.1. Under conditions}

- the matrix $L_{k} A_{k}$ in (18c) is stable

- there exists an integer $N$ and a real $\alpha>0$, such that for all $k \geq 0$

$$
\alpha I \leq \sum_{i=k}^{k+N-1} \Omega_{i+1}^{T} R_{i}^{-1} \Omega_{i+1}
$$

- the forgetting factor $\lambda \in(0,1)$

- the initial gain matrix $S_{0}$ is symmetric positive definite

then the matrix $S_{k}$ in (18i) is positive definite and bounded from above and from below by two strictly positive definite matrices for all $k \geq 0$.

A proof of this lemma can be found in Appendix A. The stability of the matrix $L_{k} A_{k}$ is directly related to the stability of the Kalman filter (12), which is assumed in this paper. In the literature the condition (26) is referred to as the persistent excitation condition (Narendra and Annaswamy (2005)).

The boundedness of $S_{k}$ ensured by the above lemma allows us to define the Lyapunov as follows

It follows

$$
V_{k}=\zeta(k)^{T} S_{k}^{-1} \zeta(k)
$$

$$
\begin{aligned}
V_{k+1} & =\zeta(k+1)^{T} S_{k+1}^{-1} \zeta(k+1) \\
& =\lambda \zeta(k)^{T}\left(I-\Theta_{k+1} \Omega_{k+1}\right)^{T} S_{k}^{-1} \zeta(k) \\
& =\lambda V_{k}-\lambda \zeta(k)^{T} \Omega_{k+1}^{T} \Theta_{k+1}^{T} S_{k}^{-1} \zeta(k) \\
& =\lambda V_{k}-\lambda\left[\Omega_{k+1} \zeta(k)\right]^{T} \Gamma_{k+1}\left[\Omega_{k+1} \zeta(k)\right]
\end{aligned}
$$

where $\Gamma_{k+1}$ is the positive definite matrix given by (18h) i.e $\left[\Omega_{k+1} \zeta(k)\right]^{T} \Gamma_{k+1}\left[\Omega_{k+1} \zeta(k)\right] \geq 0$.

We then conclude that $V_{k+1} \leq \lambda V_{k}$. This means that for any $\lambda \in(0,1), V_{k}$ decreases exponentially and that $\zeta_{k}$ and thus $\tilde{\theta}_{k}$ also tends exponentially to zero.

It then follows from (22) that, in the noise-free case,

$$
\left\{\begin{array}{l}
\lim _{k \rightarrow+\infty} z_{k}=0 \\
\lim _{k \rightarrow+\infty} \tilde{\theta}_{k}=0
\end{array} \quad \Rightarrow \quad \lim _{k \rightarrow+\infty} \tilde{x}_{k}=0\right.
$$

In the noise-corrupted case, instead of ordinary convergence, the state and parameter estimation errors converge in the mean to zeros (Guyader and Zhang (2003)).

The proposed adaptive observer has two practical advantages. The gains $L_{k}$ and $K_{k}$ for state estimation and $\Theta_{k+1}$ for parameter estimation can be tuned in two steps in simulation studies. In the first step the parameter $\theta$ is assumed known, hence the tuning of $L_{k}$ and $K_{k}$ is like in the case of the classical Kalman filter for state estimation and afterward, in the second step, $\Theta_{k+1}$ is tuned while the tuning of $L_{k}$ and $K_{k}$ is fixed. Another advantage of the adaptive observer is that the recursive computations of the gains for state and parameter estimations are separated, implying a lower numerical cost, compared to the fully coupled gain matrix computation in the Kalman filter applied to the augmented system.

\section{NUMERICAL EXAMPLE}

In this example, we use the adaptive observer to monitor faults in a system modeled by (5) with the following data

$$
\begin{aligned}
& E_{k+1}=\left[\begin{array}{cc}
0 & 0 \\
-1 & \frac{k}{k+1}
\end{array}\right], A_{k}=\left[\begin{array}{cc}
1 & -\frac{k+1}{k+0.5} \\
0 & \frac{1}{k+1}
\end{array}\right] \\
& B_{k}=\left[\begin{array}{l}
1 \\
1
\end{array}\right], \quad u(k)=\left[\begin{array}{c}
1-\frac{e^{-0.018 k}}{0.8} \sin (0.03 k) \\
1+e^{-0.03 k} \sin (0.1 k)
\end{array}\right] \\
& C_{k+1}=\left[\begin{array}{ll}
0 & 1
\end{array}\right], \bar{\theta}=1, \Phi_{k}=\left[\begin{array}{c}
1-e^{\frac{-k}{30}} \sin (0.1 k) \\
1-e^{\frac{-k}{15}} \sin (0.5 k)
\end{array}\right], \Psi_{k}=0
\end{aligned}
$$

It is easy to verify that the matrix $E_{k+1}$ is not invertible and that the matrix

$$
\left[\begin{array}{l}
E_{k+1} \\
C_{k+1}
\end{array}\right]
$$

is full column rank for all $k \geq 0$. The sampling period for the discrete time model is $1 \mathrm{~s}$ and the simulation is performed during 1000s. We simulate a degradation of the parameter $\theta$ starting from time instant $k=200$. Centered Gaussian white noise is added to the output. The simulated output with noise $y$ is plotted in figure 1 . The simulated and estimated parameter (fault) are shown in figure 2, where the dotted lines represent the true simulated parameter values, and the solid lines represent the estimated values. The estimated states are compared with the simulated states in figures 3 and 4 , where dotted line represents the true simulated state variables, and the solid line represents the estimated values. These results show that, after the transient time of about 100s corresponding to the transient time (unmodeled transient) of $\theta$, the parameter estimates follow closely the evolution of the simulated parameter. It is then possible to detect the simulated faults and estimate their severity.

\section{CONCLUSION}

This paper has dealt with fault diagnosis in descriptor systems. We have focused our study on the diagnosis of faults modeled as parameter changes in a class of discretetime descriptor systems. Under realistic assumptions on the system, a Kalman filtering algorithm is derived. Then, the adaptive observer technique is used to accomplish the fault diagnosis task. We have shown that the adaptive 


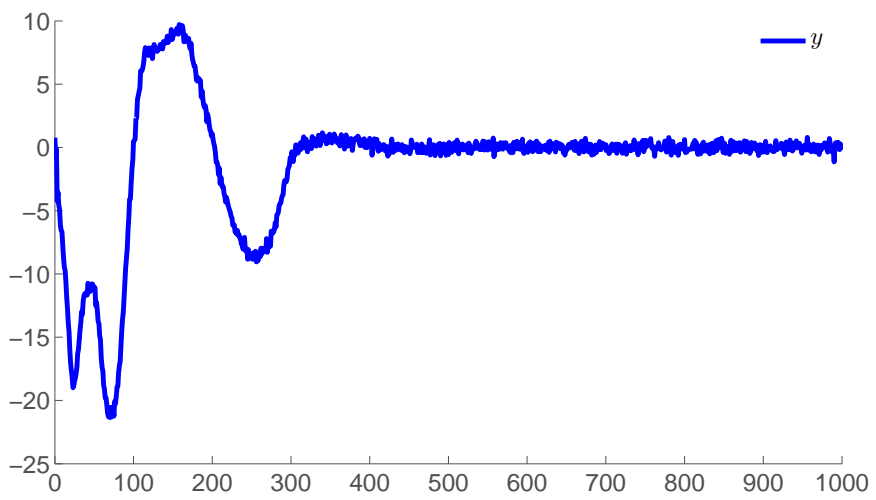

Figure 1. Graphical representations of the simulated noised output $y$ over time $(\mathrm{k})$.

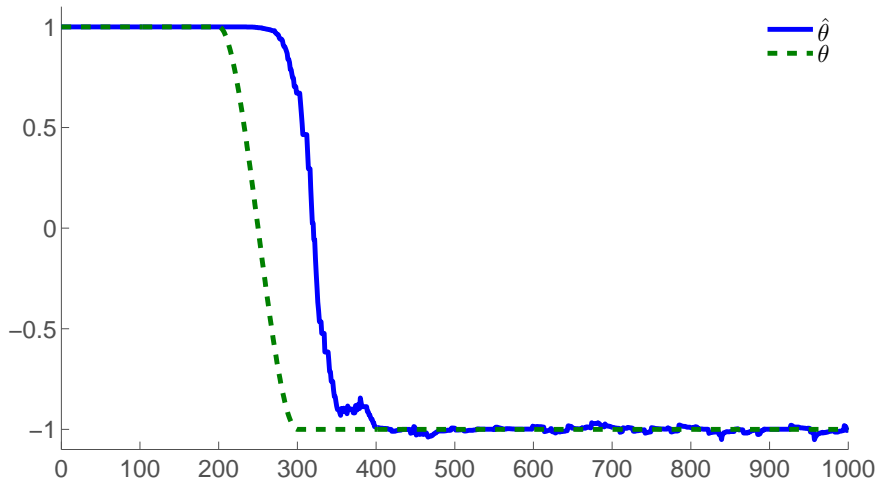

Figure 2. Graphical representations of the simulated parameter $\theta$ in dotted line and its estimate $\hat{\theta}$ in solid line, over time $(\mathrm{k})$.

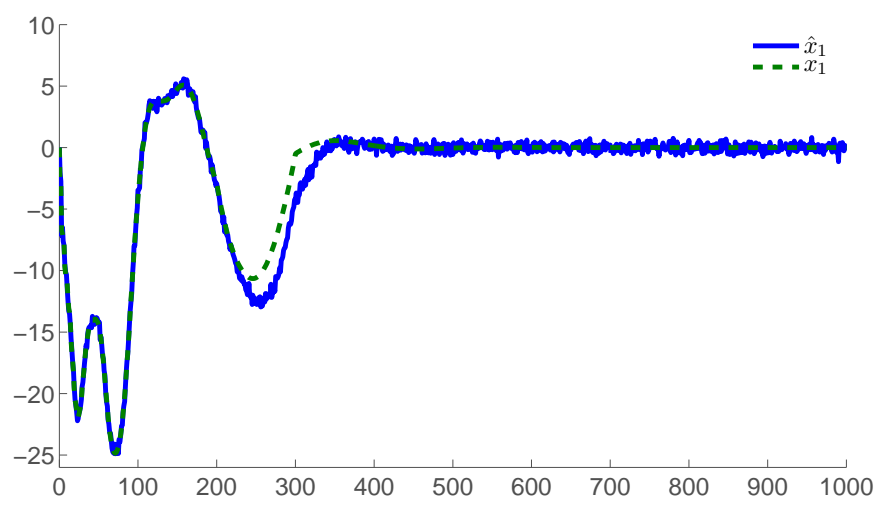

Figure 3. Graphical representations of the simulated state $x_{1}$ in dotted line and its estimate in solid line, over time $(\mathrm{k})$.

observer initially developed for explicit linear state space systems can be extended to general linear descriptor systems. The decision for fault diagnosis is based on the time evolution of parameter estimates. Simulation results are produced to illustrate the ability of the proposed approach to detect faults.

\section{REFERENCES}

Benveniste, A., Basseville, M., El Ghaoui, L., Nikoukhah, R., and Willsky, Alan, S. (1993). On the use of

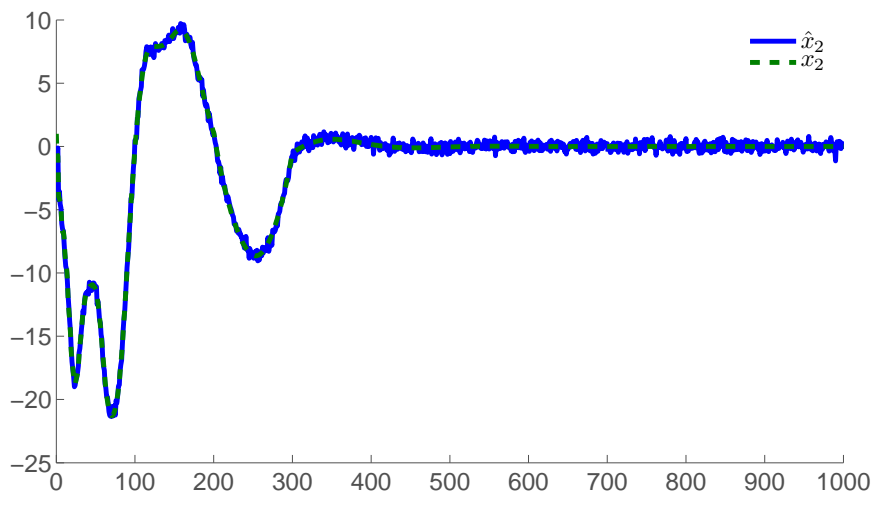

Figure 4. Graphical representations of the simulated state $x_{2}$ in dotted line and its estimate in solid line, over time $(\mathrm{k})$.

descriptor systems for failure detection and isolation. In Proceedings of the 12th IFAC World Congress, 499-502. Sydney, AU.

Campbell, S.L. and Terrell, W.J. (1991). Observability of linear time-varying descriptor systems. SIAM J. Matrix Anal. Appl., 12(3), 484-496.

Cox, H. (1964). On the estimation of state variables and parameters for noisy dynamic systems. IEEE Trans. on Automatic Control, 9(1), 5-12.

Darouach, M. and Boutayeb, M. (1995). Design of observers for descriptor systems. IEEE Trans. on Automatic Control, 40(7), $1323-1327$.

Ding, S.X. (2008). Model-Based Fault Diagnosis Techniques - Design Schemes Algorithms and tools. SpringerVerlag.

Duan, G.R., Howe, D., and Patton, R.J. (2002). Robust fault detection in descriptor linear systems via generalized unknown input observers. Int. J. of Systems Science, 33(5), 369-377.

Farza, M., M'Saada, M., Maatouga, T., and Kamounb, M. (2009). Adaptive observers for nonlinearly parameterized class of nonlinear systems. Automatica, 45(10), 2292-2299.

Guyader, A. and Zhang, Q. (2003). Adaptive observer for discrete time linear time varying systems. In 13th IFAC/IFORS Symposium on System Identification (SYSID). Rotterdam.

Isermann, R. (2006). Fault-Diagnosis System. Springer, Berlin.

Kalman, R.E. (1960). A new approach to linear filtering and prediction problems. Transaction of the ASME Journal of Basic Engineering, 1, 35-45.

Koenig, D. and Mammar, S. (2002). Design of proportional-integral observer for unknown input descriptor systems. IEEE Trans. on Automatic Control, 47(12), $2057-2062$.

Kreindler, E. and Sarachik, P. (1964). On the concepts of controllability and observability of linear systems. IEEE Trans. on Automatic Control, 9(2), 129 - 136.

Li, X., Zhang, Q., and Su, H. (2011). An adaptive observer for joint estimation of states and parameters in both state and output equations. Int. J. of Adaptive Control and Signal Processing, 25(9), 831-842.

Marx, B., Koenig, D., and Georges, D. (2004). Robust fault-tolerant control for descriptor systems. IEEE 
Trans. on Automatic Control, 49(10), 1869 - 1876.

Narendra, K. and Annaswamy, A. (2005). Stable Adaptive Systems. Dover Publications.

Nikoukhah, R., Willsky, A., and Levy, B. (1992). Kalman filtering and riccati equations for descriptor systems. IEEE Trans. on Automatic Control, 37(9), 1325 -1342.

Nikoukhah, R., Willsky, A.S., and Levy, B.C. (1987). Generalized riccati equations for two-point boundaryvalue descriptor systems. In 26th IEEE Conference on Decision and Control, 198\%., volume 26, 1140 -1141.

Polycarpou, M.M., Vemuri, A.T., and Ciric, A.R. (1997). Nonlinear fault diagnosis in differential algebraic systems. In In Proceedings of IFAC Symposium on Fault Detection, Supervision and Safety for Technical Processes, 510-515.

Shields, D.N. (1997). Observer design and detection for nonlinear descriptor systems. Int. J. of Control, 67(2), $153-168$.

Silverman, L. and Meadows, H. (1967). Controllability and observability in time-variable linear systems. SIAM Journal on Control, 5(1), 64-73.

Vemuri, A., Polycarpou, M., and Ciric, A. (2001). Fault diagnosis of differential-algebraic systems. Systems, Man and Cybernetics, Part A: Systems and Humans, IEEE Transactions on, 31(2), $143-152$.

$\mathrm{Xu}, \mathrm{A}$. and Zhang, Q. (2004). Nonlinear system fault diagnosis based on adaptive estimation. Automatica, 40(7), 1181-1193.

Zhang, Q. (2002). Adaptive observer for multiple-inputmultiple-output (mimo) linear time-varying systems. IEEE Trans. on Automatic Control, 47(3), 525 -529.

Zhang, Q., Basseville, M., and Benveniste, A. (1998). Fault detection and isolation in nonlinear dynamic systems: A combined input-output and local approach. Automatica, 34(11), 1359-1373.

Zhang, Q. and Besançon, G. (2008). An adaptive observer for sensor fault estimation in a class of uniformly observable nonlinear systems. Int. J. of Modelling, Identification and Control, 4(1), $37-43$.

Appendix A. PROOF OF LEMMA 3.1

Consider $M_{k}$ governed by

$$
\left\{\begin{array}{l}
M_{k+1}=\lambda M_{k}+\Omega_{k+1}^{T} R_{k}^{-1} \Omega_{k+1} \\
M_{0}=S_{0}^{-1}>0
\end{array}\right.
$$

Let us first study the upper bound of $M_{k}$. Due to the stability of $L_{k} A_{k}$, the matrix $\Omega_{k}$ computed from bounded $\Phi_{k}$ and $\Psi_{k}$ is also bounded. As $0<\lambda<1$, the iterations in (A.1) is exponentially stable. Hence $M_{k}$ driven by the bounded term $\Omega_{k+1}^{T} R_{k}^{-1} \Omega_{k+1}$ is also bounded.

Now consider the lower bound of $M_{k}$. A recursive evaluation of (A.1) leads to

$$
\begin{aligned}
M_{k+1} & =\lambda^{k+1} M_{0}+\sum_{j=0}^{k} \lambda^{k-j} \Omega_{j+1}^{T} R_{j}^{-1} \Omega_{j+1} \\
M_{k+1} & \geq \lambda^{k+1} M_{0}+\sum_{i=1}^{\left\lfloor\frac{k}{N}\right\rfloor} \sum_{j=(i-1) N}^{i N-1} \lambda^{k-j} \Omega_{j+1}^{T} R_{j}^{-1} \Omega_{j+1} \\
& \geq \lambda^{k+1} M_{0}+\sum_{i=1}^{\left\lfloor\frac{k}{N}\right\rfloor} \lambda^{k-i N+1} \sum_{j=(i-1) N}^{i N-1} \Omega_{j+1}^{T} R_{j}^{-1} \Omega_{j+1}
\end{aligned}
$$

where $\lfloor\cdot\rfloor$ denotes the floor (greatest integer) function.

It follows from (26) that

$$
M_{k+1} \geq \lambda^{k+1} M_{0}+\alpha\left(\sum_{i=1}^{\left\lfloor\frac{k}{N}\right\rfloor} \lambda^{k-i N+1}\right) I
$$

Then, we can conclude that $M_{k}$ is bounded from below by a strictly positive definite matrix. Hence we have proved that $M_{k}$ has both an upper bound and a strictly positive definite lower bound.

From (A.1) the inverse of the matrix $M_{k+1}$ writes

$$
M_{k+1}^{-1}=\frac{1}{\lambda}\left(M_{k}+\frac{1}{\lambda} \Omega_{k+1}^{T} R_{k}^{-1} \Omega_{k+1}\right)^{-1}
$$

By using the matrix inversion lemma

$$
(A+B C D)^{-1}=A^{-1}-A^{-1} B\left(D A^{-1} B+C^{-1}\right)^{-1} D A^{-1}
$$

it easy to check that $S_{k}=M_{k}^{-1}$ satisfies

$$
S_{k+1}=\frac{1}{\lambda}\left(I-\Theta_{k+1} \Omega_{k+1}\right) S_{k}
$$

with the initial condition $S_{0}$.

Therefore, we conclude that $S_{k}$ is bounded from above and from below by two strictly positive definite matrices, for all $k \geq 0$. 